\title{
American Academy of Periodontology
}

National Cancer Institute

\section{Source}

National Cancer Institute. American Academy of Periodontology. NCI Thesaurus. Code C63712.

Association of dental professionals specializing in the prevention, diagnosis and treatment of diseases affecting the gums and supporting structures of the teeth and in the placement and maintenance of dental implants and dedicated to advocate, educate, and set standards for advancing the periodontal and general health of the public and promoting excellence in the practice of periodontics. 